\title{
31. Lien biologie du vieillissement cancer et maladies cardiovasculaires
}

C Springer-Verlag France 2010

\section{3}

\section{Télomères et athérosclérose et vieillissement artériel \\ A. Bénétos \\ CHU de Nancy, France}

Les télomères sont des éléments essentiels qui protègent les terminaisons linéaires des chromosomes. Ces séquences répétitives non codantes d'ADN (TTAGGG) placées aux extrémités des chromosomes, jouent un rôle capital dans la capacité réplicative cellulaire et la stabilité des chromosomes. À la naissance, la longueur des télomères est génétiquement déterminée, puis elle diminue avec l'âge. Cette réduction est due au fait que pendant la réplication cellulaire l'ADN de la partie extrême des chromosomes n'est pas répliquée, ce qui conduit à une érosion (attrition) des télomères. Une reverse transcriptase, la télomérase, est capable de rajouter des séquences TTAGGG aux extrémités des chromosomes et ainsi contrebalancer l'attrition des télomères. La télomérase est active sur des cellules embryonnaires et sur des cellules germinales. Les cellules somatiques adultes en culture montrent une faible activité de télomérase.

Le raccourcissement des télomères a des conséquences sur l'intégrité et la stabilité des chromosomes, et sur la capacité réplicative cellulaire ; le résultat final est l'arrêt de la réplication cellulaire et la mort cellulaire. Par conséquent, la longueur des télomères est à la fois un indicateur du « vécu » de la cellule et de son devenir. Chez l'homme, la longueur des télomères est considérée comme un indice de l'âge biologique, et bien que cet âge biologique soit en grande partie lié à l'âge chronologique, d'autres facteurs peuvent accélérer la réduction de la taille des télomères. Le facteur le plus puissant de la perdition des bases d'ADN par réplication est le niveau du stress oxydatif. Ainsi, à un âge donné, la taille des télomères dépend essentiellement de leur taille à la naissance et des effets chroniques du stress oxydant.

Les relations entre la taille des télomères et le vieillissement artériel et l'athérosclérose sont actuellement étudiées par plusieurs équipes. Nous avons montré que la longueur des télomères était inversement corrélée avec l'augmentation de la rigidité artérielle et l'athérosclérose carotidienne. De plus, les télomères courts chez les diabétiques de type 2 augmentent le risque de microalbuminurie signe également d'attente microvasculaire au niveau rénal. D'autres équipes ont montré que les sujets coronariens et les sujets atteints d'une démence d'origine vasculaire présentaient des télomères plus courts, et que le raccourcissement des télomères était associé à la dysfonction endothéliale et le processus athéromateux. Enfin, la présence de télomères courts augmente le risque de mortalité cardiovasculaire indépendamment des autres facteurs de risque.

L'étude des mécanismes de la régulation des télomères et des conséquences cellulaires de leur raccourcissement, peut ouvrir de nouvelles voies dans la prise en charge des maladies liées au vieillissement, notamment du vieillissement artériel. 